\title{
Possible Application of X-ray Optical Elements for Reducing the Spectral Bandwidth of an X-ray SASE FEL
}

\author{
J. Feldhaus ${ }^{a}$, E.L. Saldin ${ }^{b}$, J.R. Schneider ${ }^{a}$ E.A. Schneidmiller ${ }^{b}$, \\ M.V. Yurkov ${ }^{\mathrm{c}}$ \\ ${ }^{a}$ Hamburger Synchrotronstrahlungslabor (HASYLAB) at Deutsches \\ Elektronen-Synchrotron (DESY), Notkestrasse 85, D-22607 Hamburg, Germany \\ b Automatic Systems Corporation, 443050 Samara, Russia \\ c Joint Institute for Nuclear Research, Dubna, 141980 Moscow Region, Russia
}

\begin{abstract}
A new design for a single pass X-ray Self-Amplified Spontaneous Emission (SASE) FEL is proposed. The scheme consists of two undulators and an X-ray monochromator located between them. The first stage of the FEL amplifier operates in the SASE linear regime. After the exit of the first undulator the electron bunch is guided through a non-isochronous bypass and the $\mathrm{X}$-ray beam enters the monochromator. The main function of the bypass is to suppress the modulation of the electron beam induced in the first undulator. This is possible because of the finite value of the natural energy spread in the beam. At the entrance to the second undulator the radiation power from the monochromator dominates significantly over the shot noise and the residual electron bunching. As a result the second stage of the FEL amplifier operates in the steady-state regime when the input signal bandwidth is small with respect to that of the FEL amplifier. Integral losses of the radiation power in the monochromator are relatively small because grazing incidence optics can be used. The proposed scheme is illustrated for the example of the $6 \mathrm{~nm}$ option SASE FEL at the TESLA Test Facility under construction at DESY. As shown in this paper the spectral bandwidth of such a two-stage SASE FEL $\left(\Delta \lambda / \lambda \simeq 5 \times 10^{-5}\right)$ is close to the limit defined by the finite duration of the radiation pulse. The average spectral brilliance is equal to $2 \times 10^{24}$ photons $/\left(\sec \times \mathrm{mrad}^{2} \times \mathrm{mm}^{2} \times 0.1 \%\right.$ bandw.) which is by two orders of magnitude higher than the value which could be reached by the conventional SASE FEL. The monochromatization of the radiation is performed at a low level of radiation power (about 500 times less than the saturation level) which allows one to use conventional X-ray optical elements (grazing incidence grating and mirrors) for the monochromator design.
\end{abstract}




\section{Introduction}

In this paper we propose a modification of a single pass X-ray SASE FEL allowing to reduce significantly the bandwidth of the output radiation. The proposed scheme consists of two undulators and an X-ray monochromator located between them (see Fig. 1). The first undulator operates in the linear regime of amplification starting from noise and the output radiation has the usual SASE properties. After the exit of the first undulator the electron is guided through a bypass and the X-ray beam enters the monochromator which selects a narrow band of radiation. At the entrance of the second undulator the monochromatic X-ray beam is combined with the electron beam and is amplified up to the saturation level.

The electron micro-bunching induced in the first undulator should be destroyed prior to its arrival at the second one. This can be achieved because of the finite value of the natural energy spread in the beam and by applying a special design of the electron bypass. At the entrance of the second undulator the radiation power from the monochromator dominates significantly over the shot noise and the residual electron bunching, so that the second stage of the FEL amplifier will operate in the steady-state regime when the input signal bandwidth is small with respect to the FEL amplifier bandwidth.

The monochromatization of the radiation is performed at a low level of radiation power which allows one to use conventional X-ray optical elements for the monochromator design. X-ray grating techniques can be used successfully down to wavelengths of several $\AA$ and at shorter wavelengths crystal monochromators could be used. Integral losses of the radiation power in the monochromator are relatively small because grazing incidence optics can be used. The proposed scheme possesses two significant advantages. First, it reveals a perspective to achieve monochromaticity of the output radiation close to the limit given by the finite duration of the radiation pulse and to increase the brightness and brilliance of the SASE FEL. Second, shot-to-shot fluctuations of the output radiation power could be reduced to less than $10 \%$ when the second undulator section operates at saturation. Since it is a single bunch scheme, it does not require any special time diagram for the accelerator operation.

In the following the proposed scheme is illustrated for the example of the $6 \mathrm{~nm}$ option SASE FEL at the TESLA Test Facility which is under construction at DESY. 


\section{Principle of operation of a two stage SASE FEL}

Effective operation of the proposed scheme assumes fulfillment of two conditions. First, the spectral bandwidth of the radiation at the entrance into the second undulator should be small with respect to the bandwidth of the FEL amplifier. Second, the input radiation power should be much larger than the effective power of shot noise in the electron beam. Under these conditions the second stage of the FEL amplifier operates in the conventional steady-state regime.

For the projects of SASE FELs developed at SLAC and DESY the value of the FEL amplifier bandwidth is about $0.2-0.5 \%$ [1-3]. The bandwidth of state-of-theart X-ray monochromators is much smaller than this value: using reflection gratings, which work well for wavelengths down to $\sim 1 \mathrm{~nm}$, a resolution exceeding $(\Delta \lambda / \lambda)_{m} \simeq$ $10^{-4}$ has been achieved. At wavelengths below a few $\AA$ crystal monochromators are used with a typical resolution of $(\Delta \lambda / \lambda)_{m} \simeq 10^{-5}$. Thus, the first condition can be fulfilled.

\subsection{Demodulation of the electron beam}

After the first undulator the electron and the radiation beams are separated. The electron beam is guided through a bypass and the X-ray beam enters the monochromator. The functions of the electron bypass consist in making the path lengths of the electron and the radiation beams equal, and in suppressing the modulation of the electron bunch produced in the first undulator.

Let us consider the simplest non-isochronous scheme of the electron bypass composed of three magnets which is symmetrical with respect to its center. The total magnetic field integral in the bypass is equal to zero. The trajectory of the electron beam in the bypass has the shape of an isosceles triangle with the base equal to $L$, the distance between the two undulators. The angle adjacent to the base, $\theta$, is considered to be small, $\theta \ll 1$, because the $\mathrm{X}$-ray beam also propagates under small angles.

We calculate the evolution of the beam modulation in the bypass within the onedimensional approximation. The phase space distribution of the particles is described by means of the distribution function $f(P, \psi, z)$ written in "energy-phase" variables $P=\left(\mathcal{E}-\mathcal{E}_{0}\right) / \mathcal{E}_{0}$ and $\psi=k_{\mathrm{w}} z+\omega(z / c-t)$, where $\mathcal{E}_{0}$ is the nominal energy of the particles, $k_{\mathrm{w}}=2 \pi / \lambda_{\mathrm{w}}$ is the undulator wavenumber and $\omega$ is the frequency of radiation.

At the entry of the bypass the distribution function has the form:

$$
f(P, \psi)_{\text {in }}=\frac{a_{1}}{2} f_{0}(P) \exp (-i \psi)+C . C .,
$$


where $f_{0}(P)$ corresponds to a Gaussian energy distribution of the particles

$$
f_{0}(P)=\frac{1}{\sqrt{2 \pi} \sigma_{\mathcal{E}}} \exp \left(-P^{2} / 2 \sigma_{\mathcal{E}}^{2}\right)
$$

Here $\sigma_{\mathcal{E}}$ is the standard deviation. Integration of the distribution function over energy provides the distribution of the beam density modulation:

$$
\int_{-\infty}^{\infty} d P f(P, \psi)_{\text {in }}=a_{1} \cos \psi .
$$

It is seen from this expression that at the entrance into the bypass the electron bunch is modulated in density with the wavenumber $k=k_{\mathrm{w}}+\omega / c$. As $k_{\mathrm{w}} \ll \omega / c$, the bunch modulation wavelength is almost identical to the radiation wavelength.

When an electron with relative energy offset $P$ passes the bypass, its phase shifts with respect to an electron with nominal energy by

$$
\Delta \psi(P)=\psi(P)-\psi(0)=\theta^{2} L P \omega / c
$$

As a result, one obtains the following expression for the distribution function at the exit of the bypass:

$$
f(P, \psi)_{\text {out }}=\frac{a_{1}}{2} f_{0}(P) \exp (-i \psi-i \Delta \psi(P))+C . C .
$$

which corresponds to a distribution of particles in real space given by:

$$
\begin{aligned}
& \frac{a_{1}}{2} \exp (-i \psi) \int_{-\infty}^{\infty} d P f_{0}(P) \exp \left(-i \theta^{2} L P \omega / c\right)+C . C .= \\
& \exp \left(-\sigma_{\mathcal{E}}^{2} \theta^{4} L^{2} \omega^{2} / 2 c^{2}\right) a_{1} \cos \psi
\end{aligned}
$$

It should be noticed that at the exit of the first undulator the electron beam is modulated also with respect to the electrons velocities. It can be shown that this velocity modulation is suppressed to the same degree as the density modulation (with an accuracy of a preexponential factor). Therefore, we can conclude that the problem of suppressing the beam modulations induced in the first undulator is solved quite naturally due to the presence of the energy spread in the electron beam. As a result, the initial modulation of the electron beam at the entrance into the second undulator is given by the shot noise only.

Let us consider the specific numerical example with $\sigma_{\mathcal{E}} \simeq 0.1 \%, \lambda \simeq 6 \mathrm{~nm}$, $\theta \simeq 1^{0}$ and $L \simeq 10^{3} \mathrm{~cm}$. According to expression (1), electron beam modulations are suppressed by a factor of $\exp (-5000000)$. 
For the illustration of the idea of suppressing the beam modulations we have used the simplest scheme of a non-isochronous bypass. In practice, the bypass should also be dispersion-free. It can be shown that also in this case strong suppression of the beam modulation is obtained.

Finally one has to notice that the effect of coherent synchrotron radiation produced by the electron beam should be taken into account in the design of the bypass in order to exclude emittance dilution [4]. The importance of this effect grows drastically with increasing bunch charge and shortening of the bunch length and could become significant for the parameters projected for SASE FELs (see Table 1).

\subsection{Optimization of a two-stage SASE FEL}

To provide effective operation of a two-stage SASE FEL, the input radiation power $P_{\mathrm{in}}^{(2)}$ at the entrance to the second undulator must exceed significantly the effective power, $P_{\text {shot }}$, of shot noise in the electron beam. As it was demonstrated in the previous section, the electron beam is demodulated after passing the bypass and the effective power of shot noise has the same value as at the entrance to the first undulator. When the power gain in the first undulator is equal to $G^{(1)}$ and the transmission factor of the monochromator is equal to $T_{\mathrm{m}}$, one can write:

$$
P_{\text {in }}^{(2)} / P_{\text {shot }}=G^{(1)} T_{m}
$$

The transmission factor $T_{\mathrm{m}}=R_{\mathrm{m}} K_{\mathrm{s}}$ is defined by the product of the integral reflection coefficient $R_{\mathrm{m}}$ of the mirrors and the dispersive element, and the coefficient $K_{\mathrm{s}}$ describing the radiation losses at the exit slit of the monochromator:

$$
K_{\mathrm{s}}=\frac{(\Delta \lambda / \lambda)_{\mathrm{m}}}{(\Delta \lambda / \lambda)_{\mathrm{SASE}}},
$$

where $(\Delta \lambda / \lambda)_{\mathrm{m}}$ is the resolution of the monochromator and $(\Delta \lambda / \lambda)_{\mathrm{SASE}}$ is the radiation bandwidth of SASE FEL at the exit of the first undulator. As a result, one obtains the following criterium for the power gain in the first undulator:

$$
\frac{P_{\mathrm{in}}^{(2)}}{P_{\text {shot }}}=\frac{G^{(1)} R_{\mathrm{m}}(\Delta \lambda / \lambda)_{\mathrm{m}}}{(\Delta \lambda / \lambda)_{\mathrm{SASE}}} \gg 1
$$

In addition, the resolution of the monochromator should satisfy the following conditions:

$$
\lambda / \pi \sigma_{\mathrm{z}}<(\Delta \lambda / \lambda)_{\mathrm{m}} \ll(\Delta \lambda / \lambda)_{\mathrm{SASE}}
$$


The lower limitation on $(\Delta \lambda / \lambda)_{\mathrm{m}}$ is due the fact that the length of the longitudinal coherence of the radiation can not be larger than the length of the electron bunch, $\sigma_{\mathrm{z}}$.

The upper limit on the power gain in the first undulator $G^{(\mathbf{1})}$ is given by the condition

$$
G^{(1)} \ll G_{\text {sat }}(\mathrm{SASE})
$$

where $G_{\text {sat }}(\mathrm{SASE})$ is the power gain of SASE FEL at saturation. This relation means that the first stage of the SASE FEL must operate in a linear high-gain regime. The reason for this requirement is as follows. The amplification process in the FEL leads to an energy modulation in the electron beam. After passing the bypass this energy modulation transforms into additional energy spread in the electron beam:

$$
\Delta \sigma_{\mathcal{E}} \simeq \rho \sqrt{G^{(1)} / G_{\mathrm{sat}}(\mathrm{SASE})}
$$

where $\rho$ is the saturation parameter $[5,6]$. For effective operation of the second stage of the FEL amplifier, this induced energy spread should be small:

$$
\sigma_{\mathcal{E}}^{2} \ll \rho^{2}
$$

which leads to condition (4).

When optimizing the two-stage FEL one should take into account significant shotto-shot fluctuations at the exit of the monochromator appearing due to the strongly structured spectrum of the SASE FEL at the exit of the first undulator (see Fig.3). This is the consequence of the fact that SASE FEL radiation consists of a sequence of wavepackets [7,8] (see Fig.2). The number of spikes in the spectrum is approximately equal to the number of wavepackets. Relative positions and amplitudes of maxima change from one shot to another. According to relation (3), the lower limit of the resolution of the monochromator is about $(\Delta \lambda / \lambda)_{\mathrm{m}} \simeq \lambda / \pi \sigma_{\mathrm{z}}$. On the other hand, the distance between maxima in the SASE FEL spectrum is also about $(\Delta \lambda / \lambda) \simeq$ $\lambda / \pi \sigma_{\mathrm{z}}$. Therefore, if the resolution of the monochromator is $(\Delta \lambda / \lambda)_{\mathrm{m}} \simeq \lambda / \pi \sigma_{\mathrm{z}}$, the shot-to-shot fluctuations of the radiation power at the exit of the monochromator are about one order of magnitude (see Fig.3). Remembering that there should be significant excess of radiation power above the shot noise, it is clear that the power gain $G^{(1)}$ in the first stage should be as high as possible within the limits defined by relation (4). Our estimations show that the level of the radiation power at the exit of the monochromator (averaged over shot-to-shot fluctuations) must at least be by two orders of magnitude higher than the effective power of shot noise. 
It is important to notice that a change of the power gain of the first undulator $G^{(1)}$ (i.e. the length of the first undulator) within the limits described above does not lead to an increase of the total length of the two undulators. An increase of the length of the first undulator increases the input power at the entrance of the second undulator, thus the saturation of output signal is achieved at a shorter length of the second undulator, and vice versa. This is a consequence of the fact that the first undulator operates in the high-gain linear regime (see, e.g. ref. [6] for more detail).

In conclusion to this section we combine all the conditions necessary and sufficient for the effective operation of two-stage SASE FEL:

$$
\begin{aligned}
& \frac{P_{\text {in }}^{(2)}}{P_{\text {shot }}}=\frac{G^{(1)} R_{\mathrm{m}}(\Delta \lambda / \lambda)_{\mathrm{m}}}{(\Delta \lambda / \lambda)_{\mathrm{SASE}}}>10^{2} \\
& \lambda / \pi \sigma_{\mathrm{z}}<(\Delta \lambda / \lambda)_{\mathrm{m}} \ll(\Delta \lambda / \lambda)_{\mathrm{SASE}}, \\
& G^{(1)} \ll G_{\text {sat }}(\mathrm{SASE})
\end{aligned}
$$

It is important to notice that increasing the power gain in the first stage leads to increasing heat load on the X-ray optical elements which could impose an upper limit on the value of $G^{(1)}$.

\section{Monochromator}

Seeding the second undulator with a narrow band of photons requires an optical system which extends the path length by exactly the same amount as the electron bypass for the full energy range of the FEL. For spatial reasons it could be built either in the same plane but on the opposite side as the magnets, or in a plane perpendicular to it. The basic requirements of the optical system are to select a narrow bandwidth of radiation and to merge it into the electron beam at the entrance of the second undulator with essentially unaltered beam size and divergence. Ideally, the monochromator should cover the full energy range of the FEL at a resolution close to the limit given by the electron bunch length.

In the vacuum ultraviolet (VUV) and soft x-ray region, i.e. between $\sim 1 \mathrm{~nm}$ and $\sim 100 \mathrm{~nm}$, reflection gratings are generally used to disperse the radiation. For higher energies, in the $\AA$ region, diffraction from single crystals of silicon, germanium, diamond and other materials is more appropriate to select a narrow band of photons. Due to continuous developments of scanning monochromators for the full VUV and $\mathrm{X}$-ray range of synchrotron radiation over the last three decades, a variety of different monochromator designs with the required energy resolution of $\sim 5 \times 10^{-5}$ are available 
and in routine user operation at many synchrotron radiation centers.

For a safe operation of the two-stage FEL it is essential to design the optical system such that its transmission is as high as possible. The additional advantage of high transmission is a correspondingly low radiation power absorbed by the optical components. As in a two-stage FEL amplifier the monochromator operates at a low level of radiation power, the peak and average power density could be reduced down to an uncritical level that one is used to on standard synchrotron radiation beamlines and that probably not even requires a special cooling of the optical elements.

\section{Numerical example}

The operation of a two-stage SASE FEL is illustrated for the $6 \mathrm{~nm}$ option of the SASE FEL which is under construction at DESY (see Table 1) [2]. For our calculations we have used the same parameters for the electron beam (peak current, energy spread and emittance) and the undulator (period, magnetic field and external focusing).

\subsection{First stage of the FEL amplifier}

The parameters of the first stage of the SASE FEL are presented in Table 2. It operates in a linear high-gain regime with a power gain $G^{(1)}=10^{5}$. This value is 1000 times less than the power gain at saturation,$G_{\text {sat }}($ SASE $) \simeq 10^{8}$ (see Table 1 ). Fluctuations of the beam current act as input signal, and the effective power of shot noise at the undulator entrance is equal to $100 \mathrm{~W}$. The output power averaged over the radiation pulse is equal to $10 \mathrm{MW}$. Spectral and temporal characteristics of the output radiation at the exit of the first stage are presented in Figs. 2 and 3. The spectral bandwidth of the output radiation is about $(\Delta \lambda / \lambda)_{\mathrm{SASE}} \simeq 0.5 \%[13]$.

\subsection{Monochromator}

The monochromator for the TTF-FEL should be able to select any energy between $\sim 50 \mathrm{eV}$ and $\sim 200 \mathrm{eV}$ with a resolution $\Delta \omega / \omega \sim 5 \times 10^{-5}$ in order to resolve the fine structure shown in Fig. 3. The optics needed to couple the radiation in and out of the monochromator would be particularly simple and symmetric if a monochromator design was chosen whose magnification would be independent of wavelength. Therefore, a Rowland circle grating monochromator appears to be ideally suited for this purpose since the magnification of the spherical grating is always unity, independent of wavelength. The specific design of F. Senf et al. [9] has the additional advantage that the distance between entrance and exit slit is constant and the directions of 
the in- and outgoing beams are fixed, leading to a straightforward design as shown schematically in Fig. 4. The focussing mirror demagnifies the photon beam cross section down to a few $\mu \mathrm{m}$ - this focus serves as the entrance slit of the monochromator. At the same time it increases the angular divergence from $\sim 20 \mu \mathrm{rad}$ to $\sim 1 \mathrm{mrad}$ in order to cover enough grooves of the grating to obtain a high energy resolution. The plane mirror - spherical grating combination allows to scan the monochromator such that entrance and exit slit are on the Rowland circle for all wavelengths. The exit slit selects a narrow photon band from the radiation dispersed by the grating. This diverging photon beam passing the exit slit is then collimated by a second curved mirror, just as the focussing mirror but with reverse beam direction. The mirrors in line with the undulators only serve to make the beam transport more flexible. One of them can also be made cylindrical to focus the beam sagitally on the second undulator. In principle, however, they could be omitted to increase the overall transmission.

A preliminary estimation of the transmission shows that a value of the order of $10 \%$ is realistic: For all mirrors we use carbon coatings and grazing angles of incidence of $\sim 4^{0}$, giving a reflectivity of $90 \%$ for each mirror. Assuming a grating efficiency of $15 \%$ and five mirrors with $90 \%$ reflectivity then gives a total transmission of nearly $9 \%$. The mirror reflectivity could even be enhanced by working at more grazing angles (see Fig. 5), the total number of mirrors used is less important. Much more critical is the grating efficiency which strongly depends on the manufacturing process, but also on the type of grating, its line density and other parameters. Efficiencies far exceeding $15 \%$ have been measured for specific gratings [10], and also a model calculation for a blazed grating with reasonable but not at all optimized parameters shows that higher efficiencies are possible in this energy range (see Fig. 6). Clearly, extensive and detailed investigations are necessary to optimize the overall performance of the optical system.

\subsection{Second stage of the FEL amplifier}

The chosen parameters for the first stage of the SASE FEL and the monochromator satisfy the conditions (5). The numerical example presented in section 2.1 showed that the beam modulation induced in the first section is totally suppressed in the bypass. Therefore, the second stage of the two-stage FEL amplifier operates in the conventional steady-state regime.

The parameters of the second stage of the SASE FEL are presented in Table 3. The average value of the input radiation power is $10 \mathrm{~kW}$, which results in a saturation length of $16 \mathrm{~m}$. The quality of the output radiation of the two-stage SASE FEL 
exceeds significantly that of the conventional SASE FEL (see Tables 3 and 1).

\subsection{Characteristics of output radiation}

Next the output characteristics of the radiation of the proposed two-stage FEL are compared with those of the conventional SASE FEL (see Tables 3 and 1).

Temporal and spectral structure of the output radiation of the conventional SASE FEL are presented in Figs.7 and 8. It is seen that the radiation pulse consists of a large number of wavepackets (spikes). While there is full coherence of the radiation within one spike, there is no phase correlation between adjacent spikes. As a result, the autocorrelation time $\tau_{1 / 2}$ (which corresponds to a decrease by a factor of two of the first order correlation function) is about $3 \mathrm{fs}$ which is by two orders of magnitude less than the total duration of the radiation pulse $[13,14]$.

The radiation bandwidth of the conventional SASE FEL is about $(\Delta \lambda / \lambda)_{\mathrm{SASE}} \simeq$ $0.5 \%$. The shape of the spectrum is not smooth but spiked as shown in Fig. 8. To perform experiments which require narrow bandwidth of the output radiation, a monochromator has to be installed at the FEL amplifier exit. In accordance with Fig. 8 the shot-to-shot fluctuations of the radiation power after this monochromator will increase with increasing resolution of the monochromator. Moreover, conventional X-ray optical elements will suffer from heat load due to the high output radiation power and probably filters have to be installed before the monochromator. As a result, the brightness and the brilliance of the FEL radiation available at the experimental station might be reduced significantly.

The characteristics of the two-stage SASE FEL operating at saturation are presented in Figs.9-14.

The dependence of the output radiation power on the undulator length of the second stage is presented in Fig.9. The output radiation power is close to that of the conventional SASE FEL while the spectral bandwidth is by two orders of magnitude narrower. Thus, the spectral brightness and brilliance of the output radiation exceed the corresponding values of a conventional SASE FEL by two orders of magnitude. In addition, longitudinal coherence of the output radiation over the full radiation pulse is obtained which leads to an autocorrelation time $\tau_{1 / 2} \simeq 300$ fs (see Fig.10). The spectral bandwidth of $\Delta \lambda / \lambda \simeq 5 \times 10^{-5}$ is close to the limit given by the finite duration of the radiation pulse.

Fig.11 shows that the value of the output radiation power is rather insensitive to the fluctuations of the input signal when the second stage of the FEL amplifier operates near the saturation point. For a length of the second undulator of $16 \mathrm{~m}$ fluctuations of the input power by one order of magnitude result in $10 \%$ fluctuations 
of the output power only. Higher stability of the output radiation power could be achieved by increasing the length of the undulator (see Fig. 11).

Fig.12 presents the frequency characteristics of the FEL amplifier, i.e. the dependence of the output power on the deviation of the frequency of the input signal. In particular, this plot allows one to obtain requirements for the level of stability of the electron beam energy [6].

In Fig.13 the distributions of the radiation power density and the beam current density at the FEL amplifier exit (at saturation point) are plotted as functions of radius. Fig.14 shows the angular distribution of the radiation power in the Fraunhofer diffraction zone. Both figures demonstrate the excellent spatial properties of the output radiation.

\section{Acknowledgement}

We thank J. Rossbach for his interest in this work. 


\section{References}

[1] R. Tatchyn, J. Arthur, M. Baltay et al., Nucl. Instrum. and Methods A375(1996)274.

[2] "A VUV Free Electron Laser at the TESLA Test Facility at DESY. Conceptual Design Report", DESY print, TESLA-FEL 95-03, Hamburg (1995).

[3] J. Rossbach, Nucl. Instrum. and Methods A375(1996)269.

[4] Ya.S. Derbenev, J. Rossbach, E.L. Saldin and V. Shiltsev, DESY Print, TESLA-FEL 95-05, Hamburg (1995).

[5] R. Bonifacio, C. Pellegrini and L. Narducci, Opt. Commun. 50(1984)373

[6] E.L. Saldin, E.A. Schneidmiller and M.V. Yurkov, Phys. Rep. 260(1995)187.

[7] R. Bonifacio, L. De Salvo, P. Pierini, N. Piovella and C. Pellegrini, Nucl. Instrum. and Methods A341(1994)181.

[8] R. Bonifacio, L. De Salvo, P. Pierini, N. Piovella and C. Pellegrini, Phys. Rev. Lett. 73(1994)70.

[9] F. Senf, F. Eggenstein, and W. Peatman, Rev. Sci. Instrum. 63(1992)1326.

[10] J.B. West and H.A. Padmore, in Handbook on Synchrotron Radiation, Vol.2, Ed. G.V. Marr (North Holland, Amsterdam, 1987), p. 21.

[11] F. Schäfers, BESSY, Berlin, private communication.

[12] B. Henke, E.M. Gullikson and J.C. David, Atomic and Nuclear Data Tables 54(1993)181.

[13] E.L. Saldin, E.A. Schneidmiller and M.V. Yurkov, DESY Print, TESLA-FEL 96-07, Hamburg(1996)

[14] P. Pierini and W. Fawley, Nucl. Instrum. and Methods A375(1996)332. 


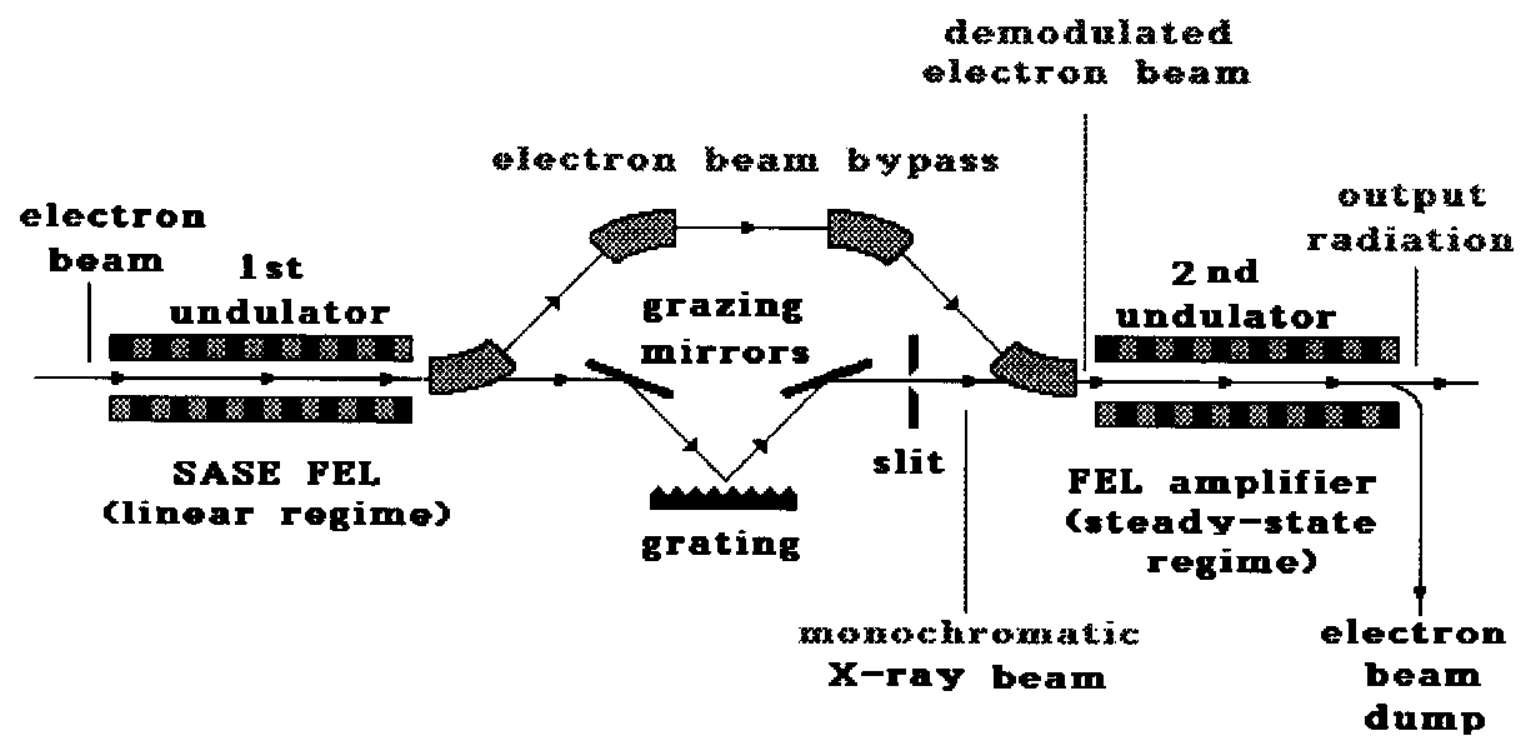

Fig. 1. The principal scheme of a single-pass two-stage SASE X-ray FEL with monochromator. 
Table 1

Parameters of the conventional SASE FEL at DESY

\begin{tabular}{|c|c|}
\hline \multicolumn{2}{|l|}{$\underline{\text { Electron beam }}$} \\
\hline Energy, $\mathcal{E}_{0}$ & $1000 \mathrm{MeV}$ \\
\hline Peak current, $I_{0}$ & $2500 \mathrm{~A}$ \\
\hline rms bunch length, $\sigma_{z}$ & $50 \mu \mathrm{m}$ \\
\hline Normalized rms emittance, $\epsilon_{\mathrm{n}}$ & $2 \pi \mathrm{mm} \mathrm{mrad}$ \\
\hline rms energy spread & $0.1 \%$ \\
\hline External $\beta$-function, & $300 \mathrm{~cm}$ \\
\hline rms transverse beam size & $57 \mu \mathrm{m}$ \\
\hline Number of bunches per train & 7200 \\
\hline Repetition rate & $10 \mathrm{~Hz}$ \\
\hline \multicolumn{2}{|l|}{$\underline{\text { Undulator }}$} \\
\hline Type & Planar \\
\hline Length of undulator, $L_{\mathrm{w}}$ & $20 \mathrm{~m}$ \\
\hline Period, $\lambda_{w}$ & $2.73 \mathrm{~cm}$ \\
\hline Peak magnetic field, $H_{\mathrm{w}}$ & $4.97 \mathrm{kGs}$ \\
\hline \multicolumn{2}{|l|}{$\underline{\text { Radiation }}$} \\
\hline Wavelength, $\lambda$ & $6.4 \mathrm{~nm}$ \\
\hline Bandwidth, $\Delta \lambda / \lambda$ & $0.5 \%$ \\
\hline rms angular divergence & $15 \mu \mathrm{rad}$ \\
\hline rms spot size at the undulator exit & $90 \mu \mathrm{m}$ \\
\hline autocorrelation time, $\tau_{1 / 2}$ & $3 \mathrm{fs}$ \\
\hline Power average over pulse & $5 \mathrm{GW}$ \\
\hline Flash energy & $1.5 \mathrm{~mJ}$ \\
\hline Average power & $100 \mathrm{~W}$ \\
\hline \multirow[t]{4}{*}{ Average spectral brightness } & $10^{21}$ \\
\hline & Phot./(sec $x$ \\
\hline & $\operatorname{mrad}^{2} x$ \\
\hline & $0.1 \%$ bandw.) \\
\hline \multirow[t]{2}{*}{ Average spectral brilliance } & $2 \times 10^{22}$ Phot. $/(\sec \times$ \\
\hline & $\operatorname{mrad}^{2} \times \mathrm{mm}^{2} \times 0.1 \%$ bandw.) \\
\hline
\end{tabular}


Table 2

Parameters of the first stage of the two-stage SASE FEL

\begin{tabular}{ll}
\hline Mode of operation & $\begin{array}{l}\text { SASE, linear } \\
\text { amplification }\end{array}$ \\
Effective power of shot noise, $P_{\text {shot }}$ & $100 \mathrm{~W}$ \\
Length of undulator, $L_{\mathrm{w}}$ & $12 \mathrm{~m}$ \\
Effective gain, $G$ & $10^{5}$ \\
Output radiation & \\
\hline Wavelength, $\lambda$ & $6.4 \mathrm{~nm}$ \\
Bandwidth, $\Delta \lambda / \lambda$ & $0.5 \%$ \\
Autocorrelation time, $\tau_{1 / 2}$ & $2 \mathrm{fs}$ \\
rms spot size at the undulator exit & $40 \mu \mathrm{m}$ \\
rms angular divergence & $18 \mu \mathrm{rad}$ \\
Peak power & $50 \mathrm{MW}$ \\
Power average over pulse, & $10 \mathrm{MW}$ \\
Flash energy & $3 \mu \mathrm{J}$ \\
Average power & $0.2 \mathrm{~W}$ \\
\hline
\end{tabular}


Table 3

Parameters of the second stage of the two-stage SASE FEL

\begin{tabular}{ll}
\hline Mode of operation & Steady-state, \\
saturation
\end{tabular}


(a)

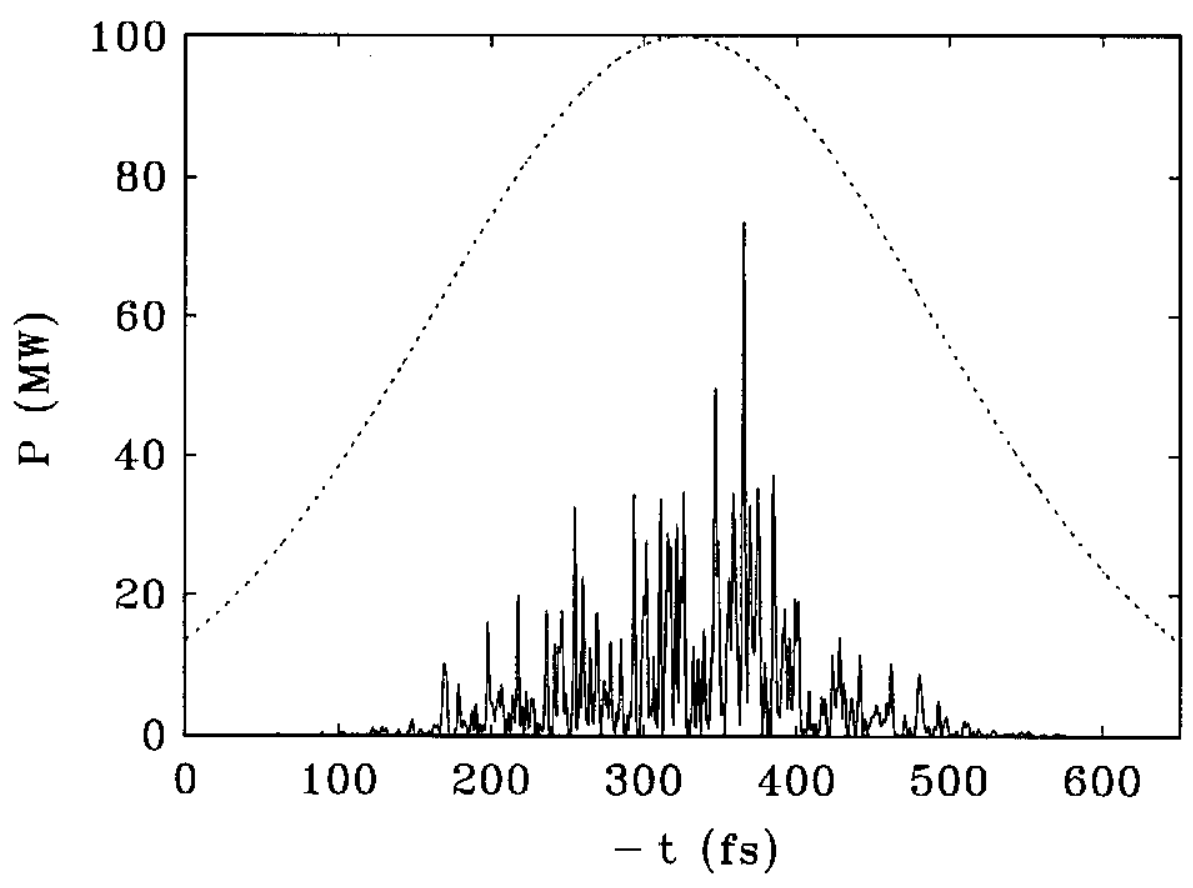

(b)

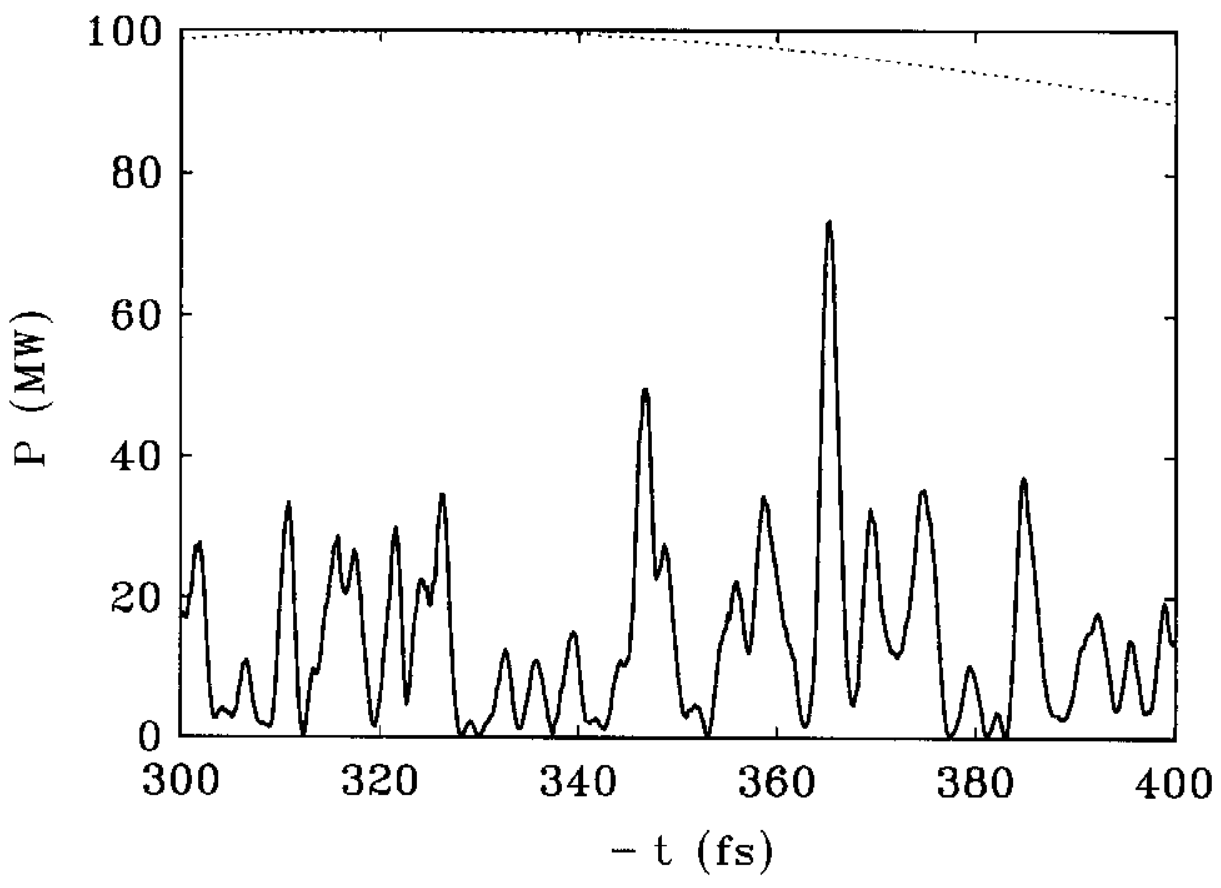

Fig. 2. Temporal structure of a radiation pulse at the exit of the first undulator. Graph (a) is a plot of the full length of the electron beam and graph (b) presents an enlarged fraction of graph (a). The dashed line presents the corresponding distribution of the electron beam current. 
(a)

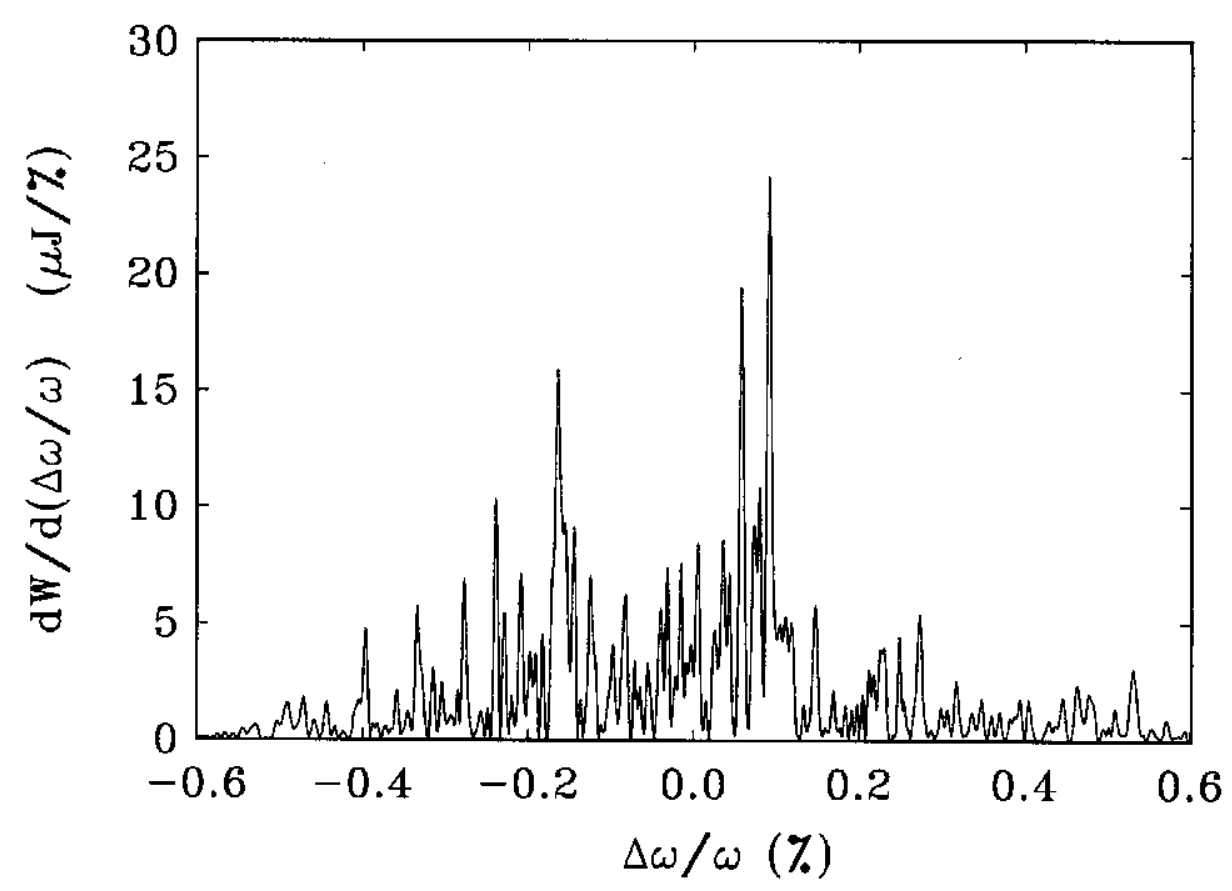

(b)

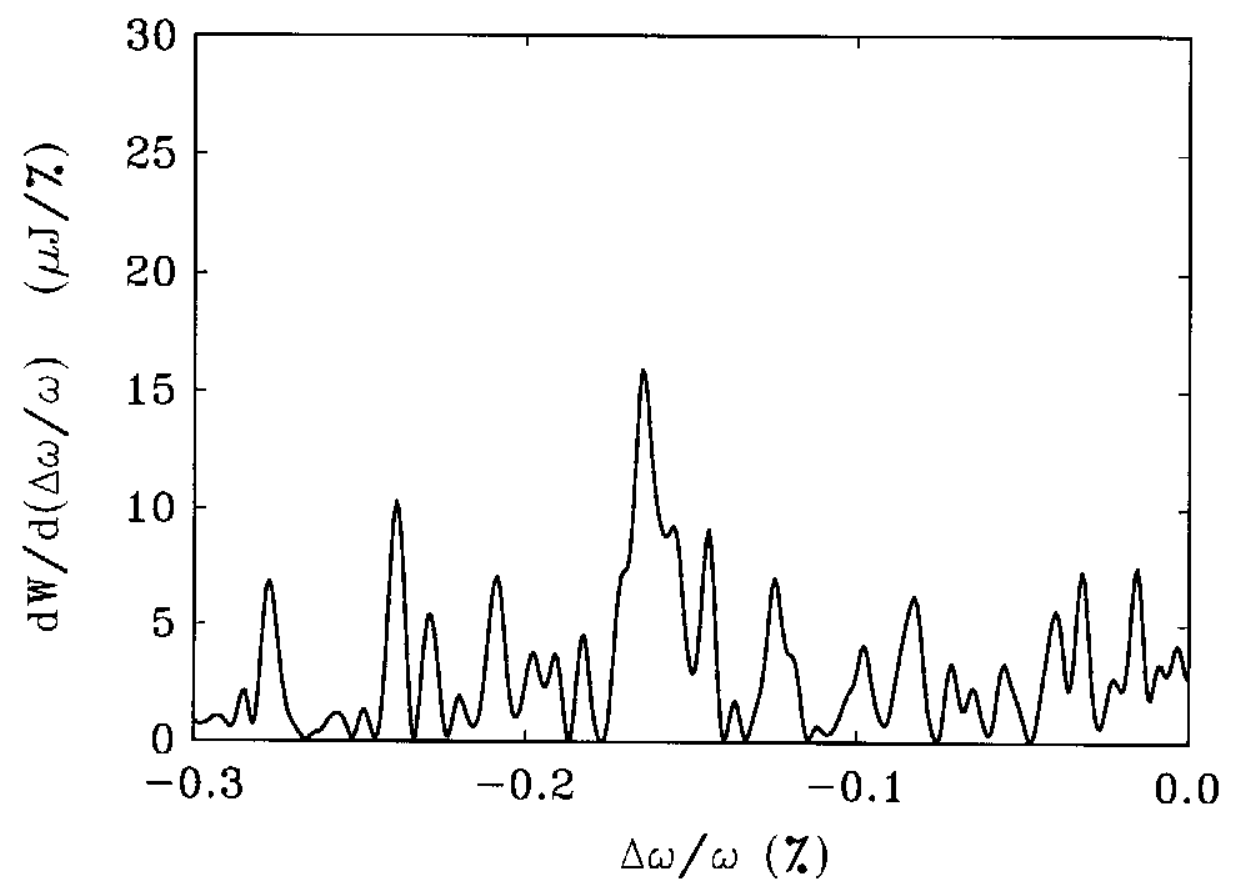

Fig. 3. Spectral distribution of the radiation pulse at the exit of the first undulator. Graph (a) is a plot of the full spectrum and graph (b) presents an enlarged fraction of graph (a). 


\section{Rowland circle monochromator}

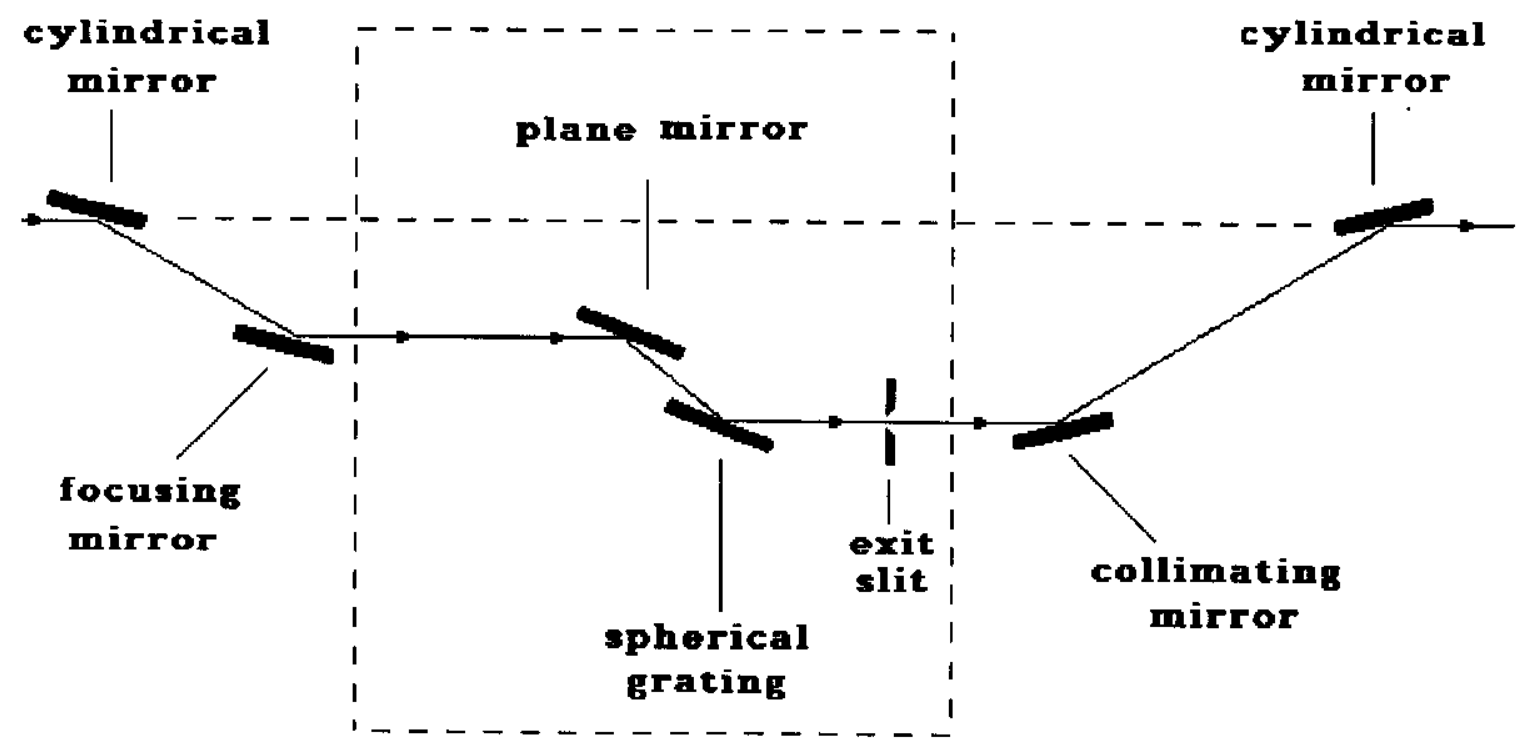

Fig. 4. Layout of a grating monochromator for the TTF FEL. 


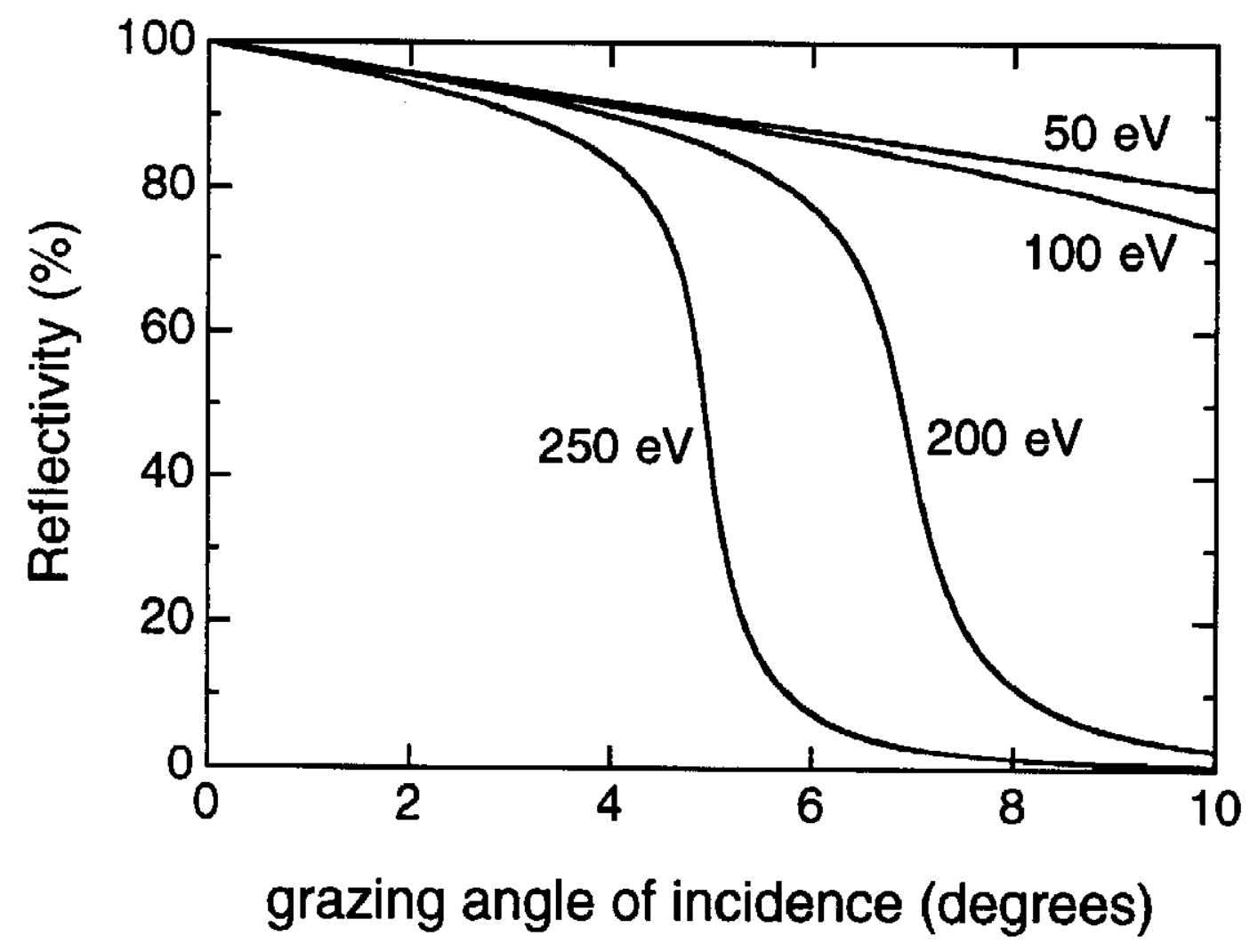

Fig. 5. Angular dependence of the reflectivity of a carbon coated mirror for different photon energies as calculated by Fresnel's equations using optical constants of Henke et al. [12]. 


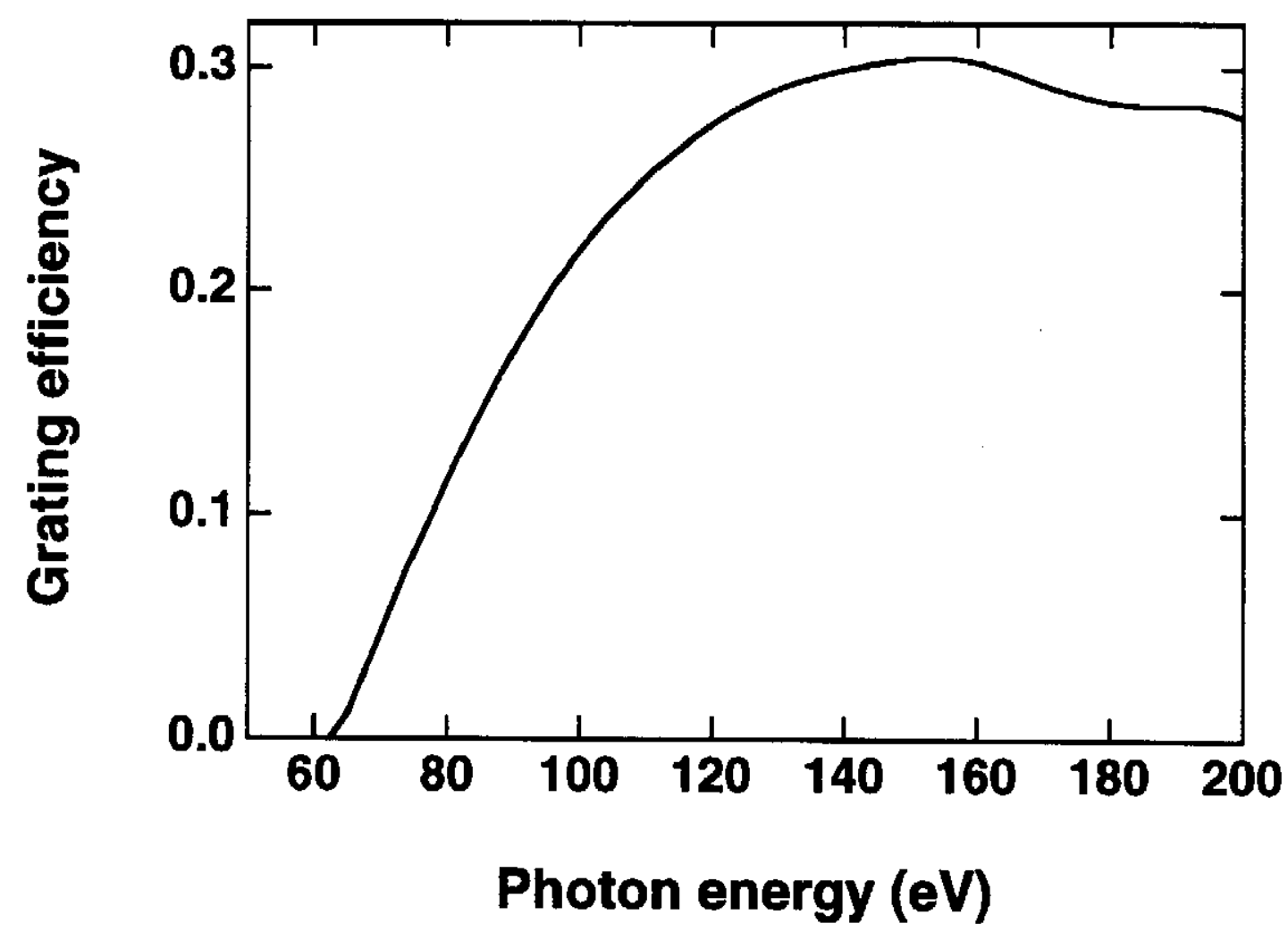

Fig. 6. Diffraction efficiency for a blazed grating calculated with REFLEC [11]. In this example the deflection angle is $2 \theta=8^{\circ}$, the blaze angle is $2^{0}$ and the line density is 500 lines per millimeter. 
(a)

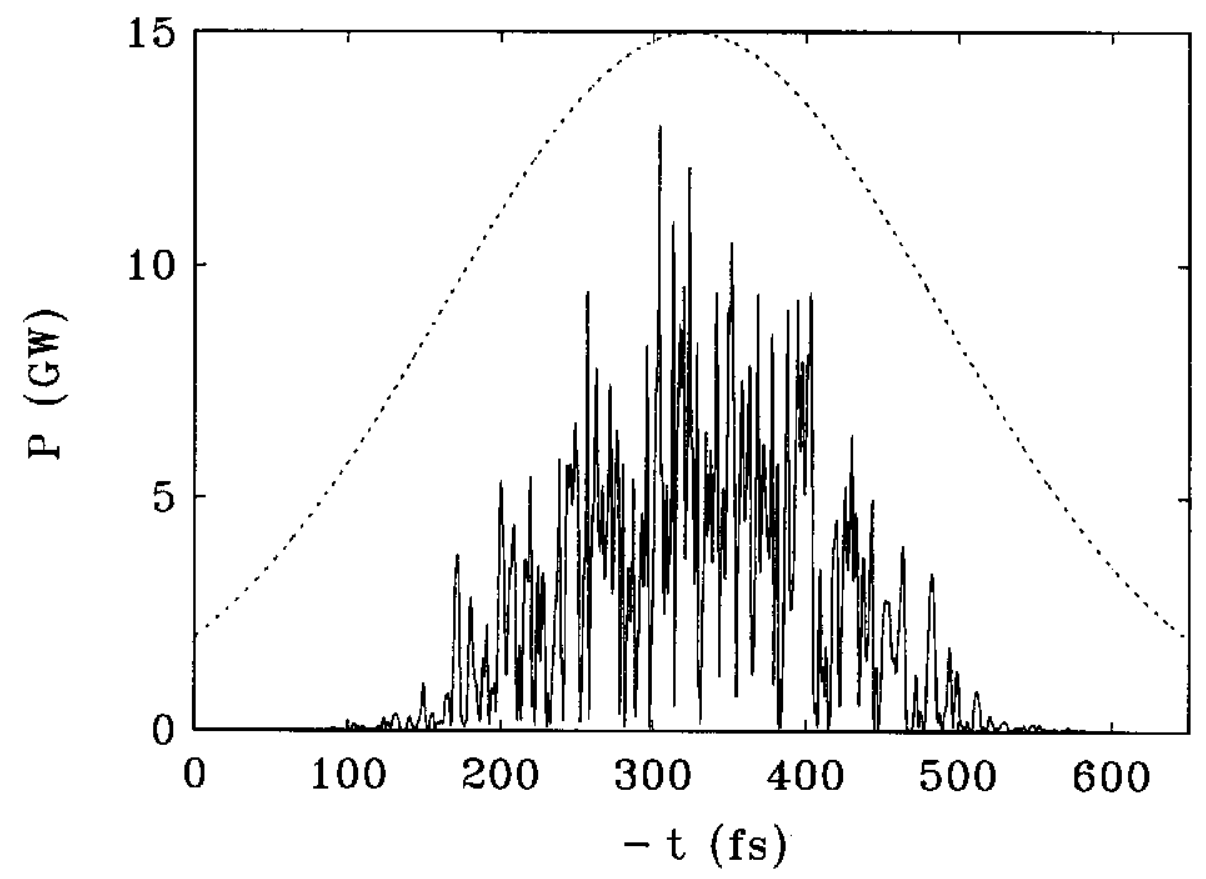

(b)

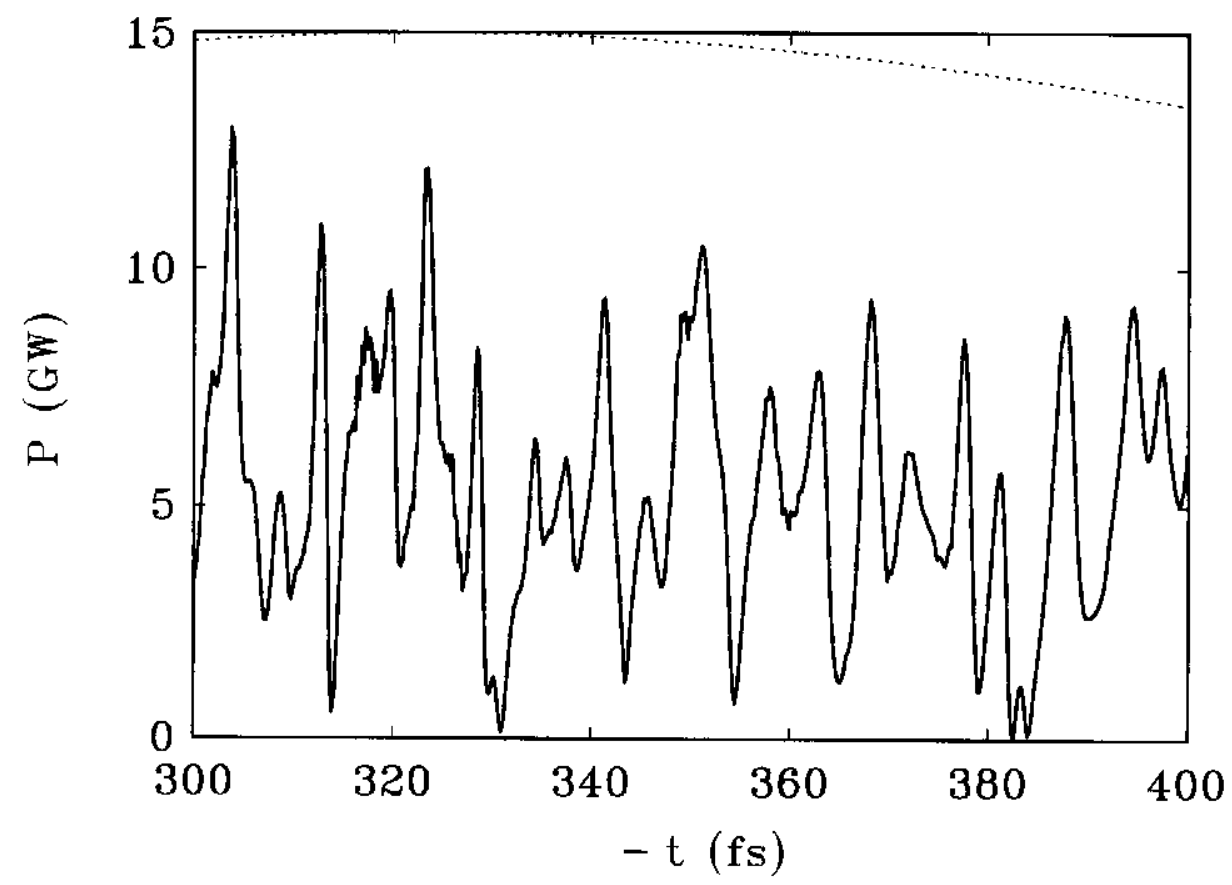

Fig. 7. Temporal structure of the radiation pulse at the saturation point of the conventional SASE FEL. Graph (a) covers the full length of the electron bunch and graph (b) presents an enlarged fraction of graph (a). The dashed line presents the corresponding distribution of the electron beam current. 
(a)

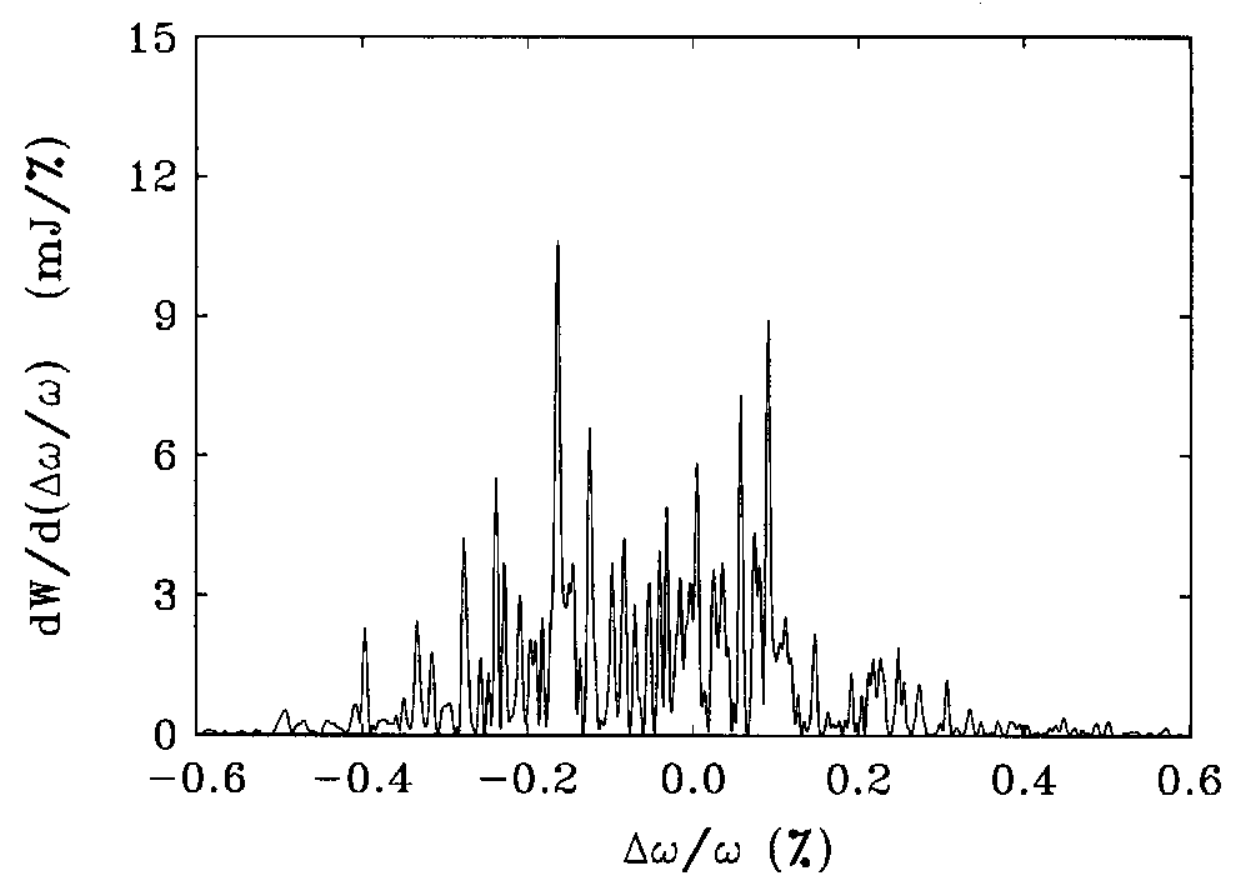

(b)

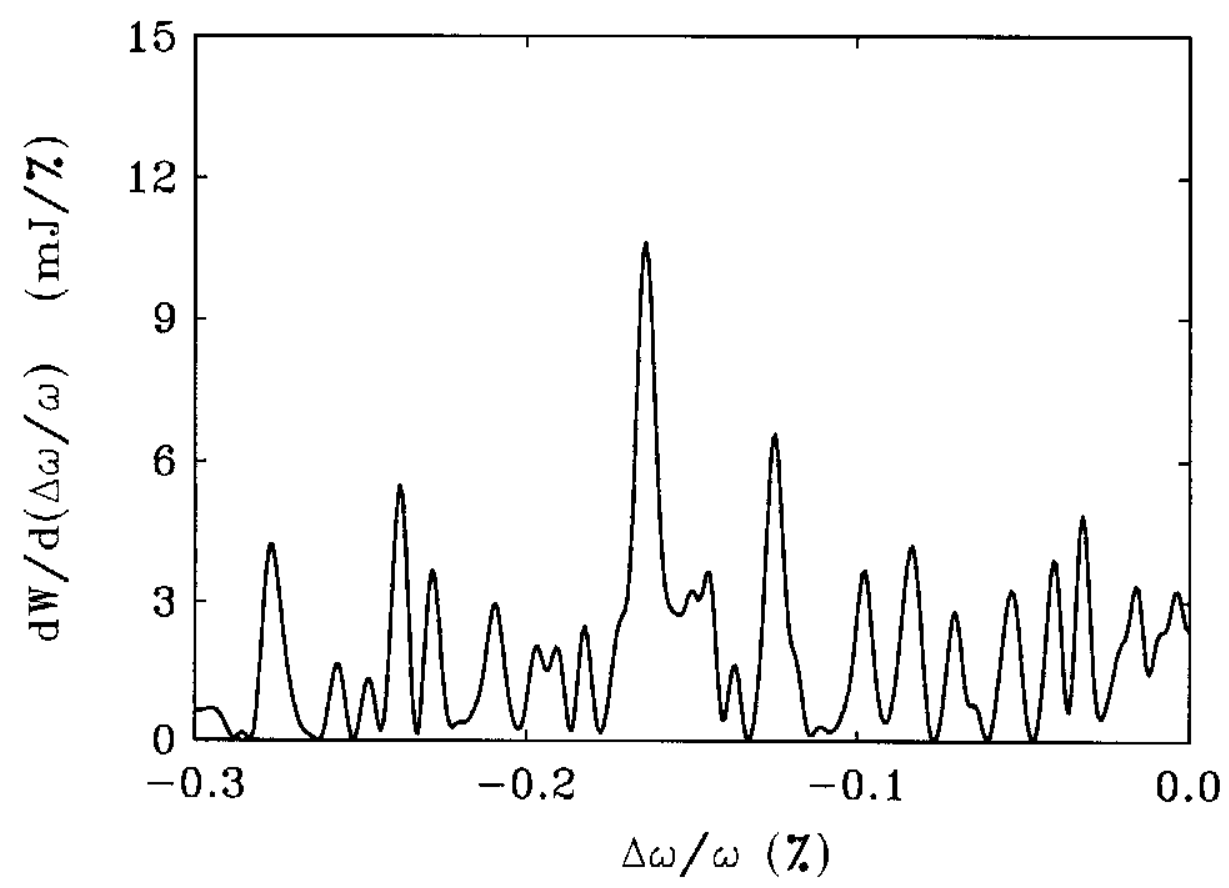

Fig. 8. Spectral distribution of the radiation pulse at the saturation point of the conventional SASE FEL. Graph (a) is a plot of the full spectrum and graph (b) presents an enlarged fraction of graph (a). 


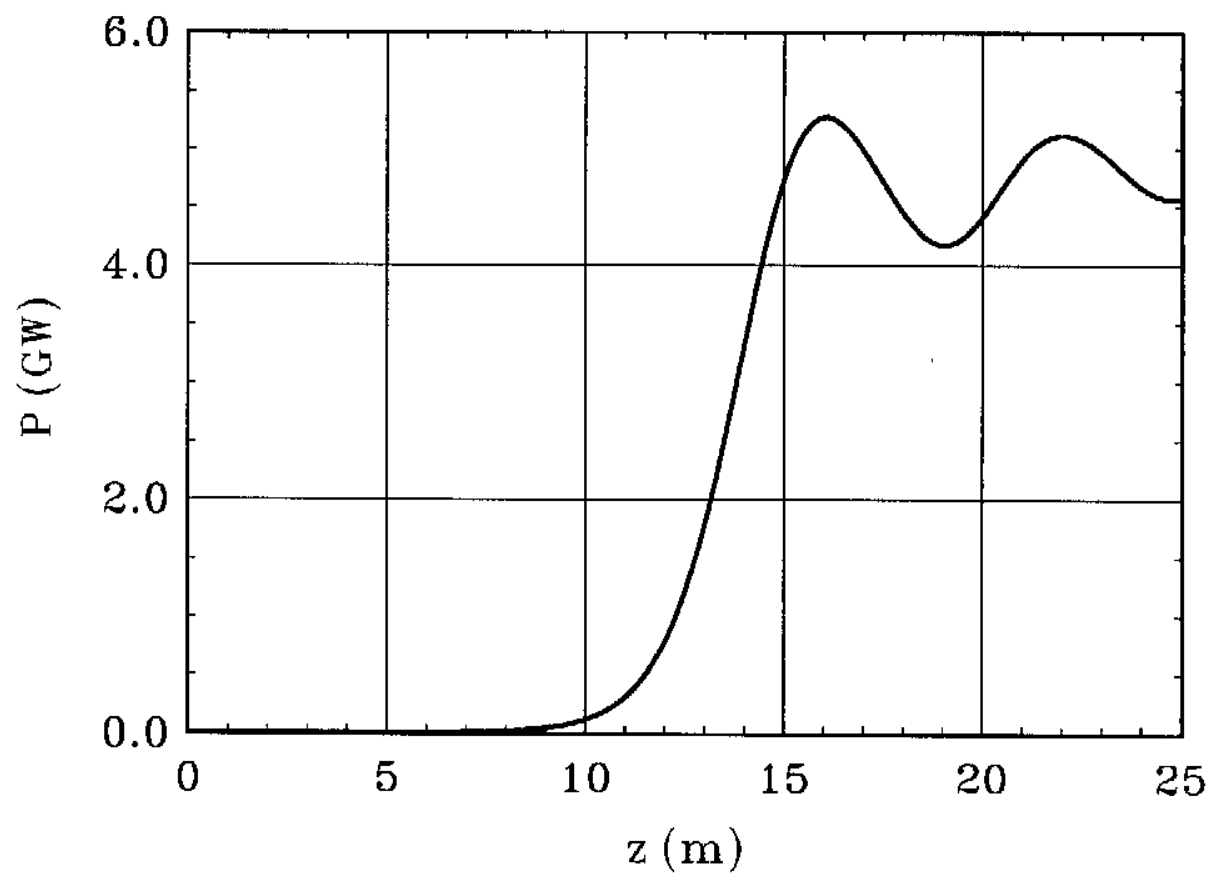

Fig. 9. Output power of the FEL amplifier operating in the steady-state regime as a function of undulator length. Input power $P_{\text {in }}=10 \mathrm{~kW}$. 


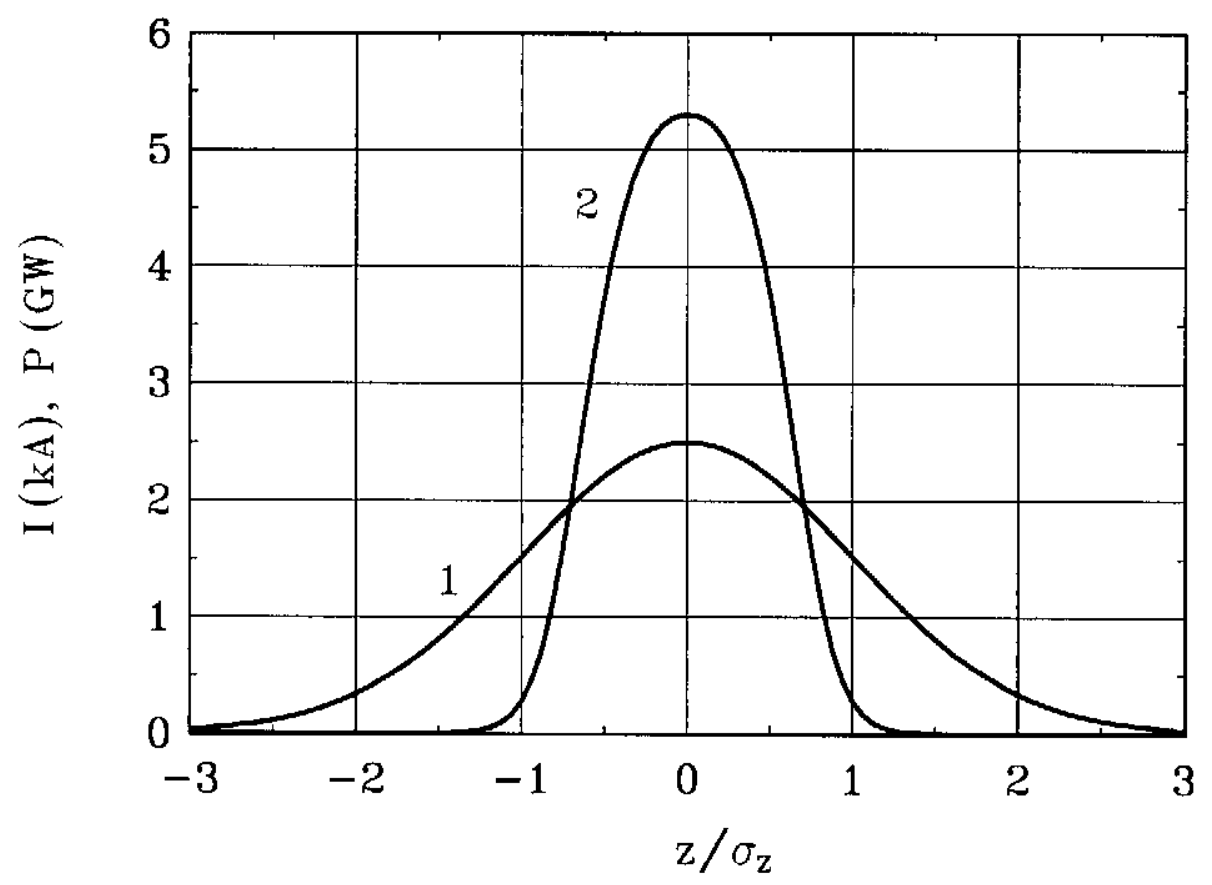

Fig. 10. Longitudinal distribution of the radiation power (2) and the electron beam current (1) at the saturation point. 


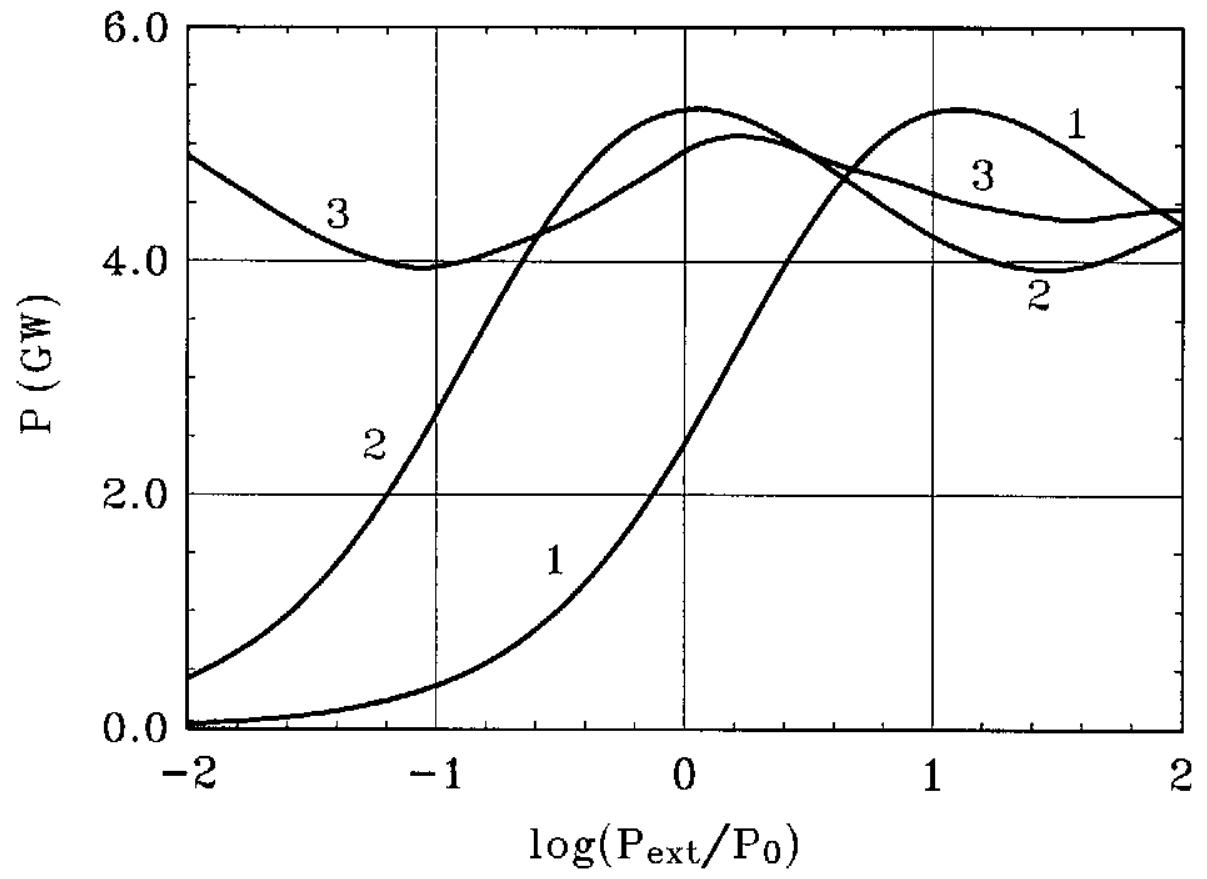

Fig. 11. Dependence of the output power on the input power for the FEL amplifier operating in the steady-state regime, (1): For an undulator length $L_{\mathrm{w}}=14 \mathrm{~m},(2): L_{\mathrm{w}}=16 \mathrm{~m},(3)$ : $L_{\mathrm{w}}=20 \mathrm{~m}$. Nominal external power $P_{0}=10 \mathrm{~kW}$. 


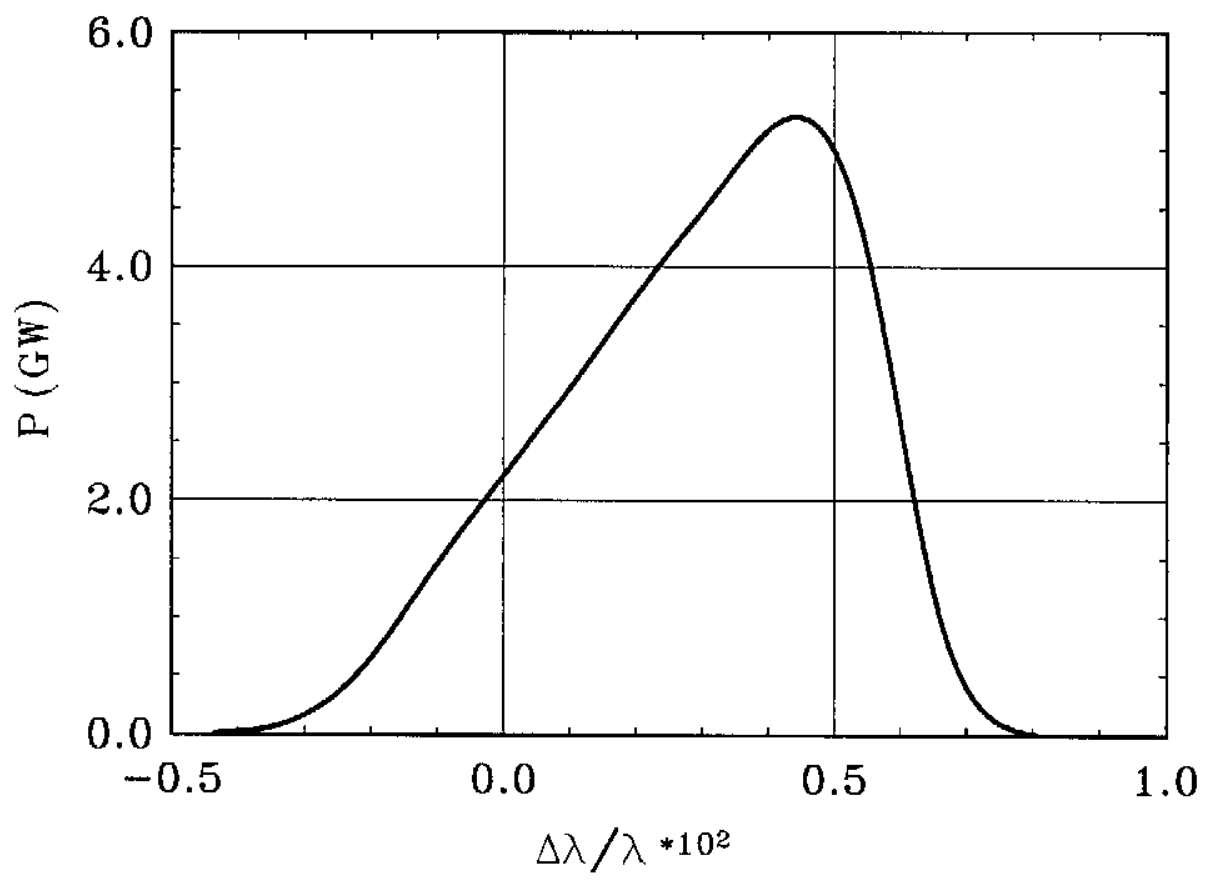

Fig. 12. Frequency characteristic of the FEL amplifier operating in the steady-state regime for an input power $P_{\text {in }}=10 \mathrm{~kW}$ and an undulator length $L_{\mathrm{w}}=16 \mathrm{~m}$ (saturation point). 


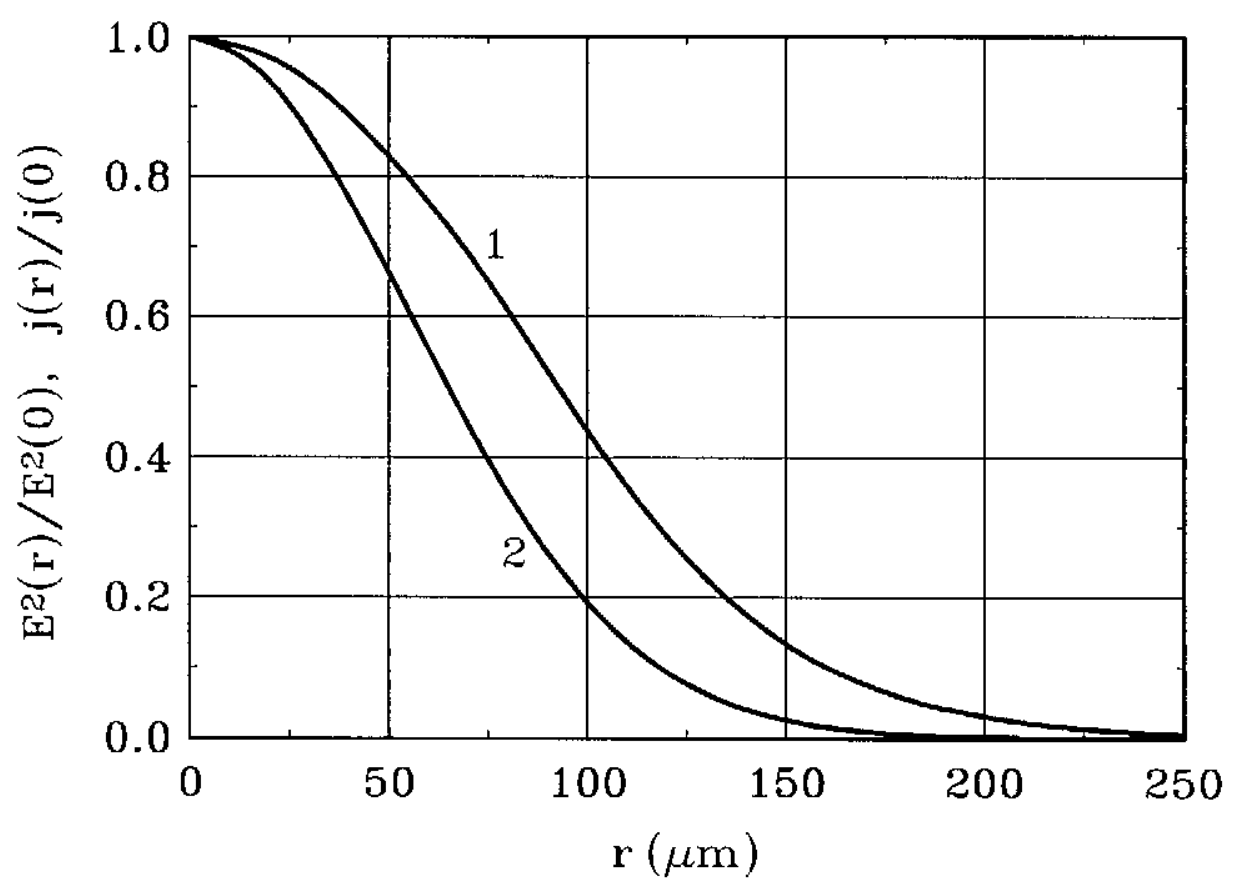

Fig. 13. Radial distribution of the radiation power density (1) and the electron beam current density (2) at the saturation point. 


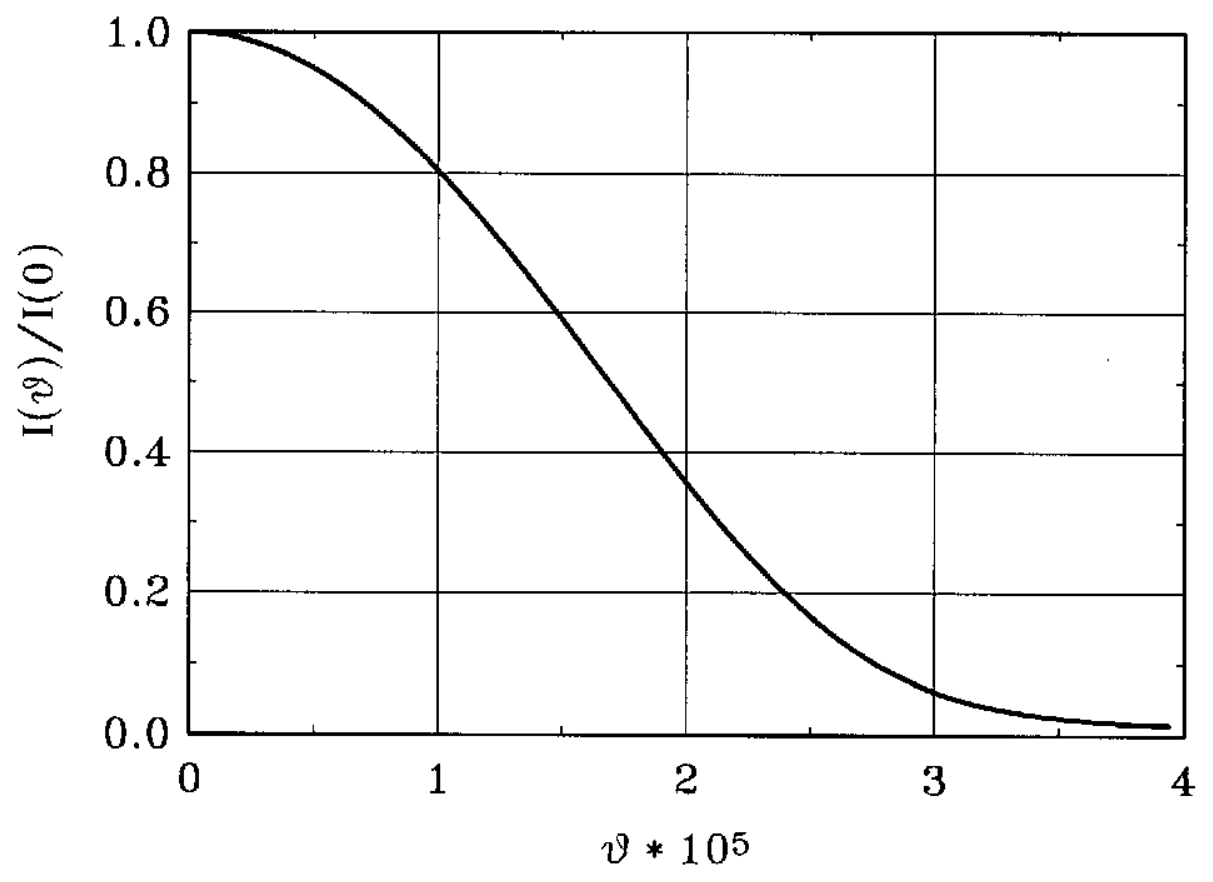

Fig. 14. Angular distribution of the radiation power in the Fraunhofer diffraction zone at the saturation point. 